\title{
A Class of Transformation Matrices and Its Applications
}

\author{
Wenhui Liu, ${ }^{1}$ Feiqi Deng, ${ }^{1}$ Jiarong Liang, ${ }^{2}$ and Haijun Liu ${ }^{3}$ \\ ${ }^{1}$ College of Automation Science and Engineering, South China University of Technology, Guangzhou 510641, China \\ ${ }^{2}$ School of Computer, Electronics and Information, Guangxi University, Nanning 530004, China \\ ${ }^{3}$ School of Mathematics and Statistics, Zhengzhou University, Zhengzhou 450001, China \\ Correspondence should be addressed to Feiqi Deng; aufqdeng@scut.edu.cn
}

Received 8 November 2013; Revised 13 February 2014; Accepted 13 February 2014; Published 1 April 2014

Academic Editor: Turgut Öziş

Copyright (C) 2014 Wenhui Liu et al. This is an open access article distributed under the Creative Commons Attribution License, which permits unrestricted use, distribution, and reproduction in any medium, provided the original work is properly cited.

\begin{abstract}
This paper studies a class of transformation matrices and its applications. Firstly, we introduce a class of transformation matrices between two different vector operators and give some important properties of it. Secondly, we consider its two applications. The first one is to improve Qian Jiling's formula. And the second one is to deal with the observability of discrete-time stochastic linear systems with Markovian jump and multiplicative noises. A new necessary and sufficient condition for the weak observability will be given in the second application.
\end{abstract}

\section{Introduction}

The vector operator is an important concept in matrix analysis and an effective mathematical tool in many applications [1]. For the symmetric case, we will consider a new vector operator. We find that there exists a class of transformation matrices between the two vector operators and they can be used to solve many problems. In order to demonstrate the importance of these transformation matrices, this paper will consider their two applications in control theory.

Qian Jiling's formula is an important and interesting formula to compute the Lyapunov functions [2]. Our paper will improve Qian Jiling's formula by the help of these transformation matrices.

Observability and detectability are two basic concepts in control theory [3]. These concepts of deterministic systems had been extended to the stochastic systems over the past few decades, such as [4-11] and references therein. Particularly, this paper will focus on the observability/detectability for discrete-time stochastic linear systems. The definition of the uniform observability/detectability [4] for deterministic discrete-time time-varying linear systems had been extended to stochastic linear systems in [5]. Actually the weak observability/detectability in [5] and the uniform observability/detectability in [4] are consistent. Reference [5] showed that the weak detectability was weaker than the mean square detectability. Still, the weak detectability plays the same role in the discussion of algebraic Lyapunov and Riccati equations. Under the same framework, [6] investigated the observability (i.e., the weak observability in [5]) problems for a class of discrete-time stochastic linear systems subject to Markovian jump and multiplicative noises and got some necessary and sufficient conditions. Reference [7] studied the equivalence between two different definitions of the observability/detectability for discrete-time stochastic linear systems. These are good works about the observability and detectability of discrete-time stochastic linear systems. However, our paper will further study the weak observability for discrete-time stochastic linear systems with the help of these transformation matrices. The system in [6] is more complicated than ours, but we get some more profound conclusions which are not included in [6]. Although the systems in [5, 7] are two special cases of our system, our results are not their parallel results. We need these transformation matrices in order to get these new results.

The outline of this paper is as follows. In Section 2, we define a class of transformation matrices which exists independently and study its important properties. Section 3 considers its applications. We use it to improve Qian Jiling's formula in Section 3.1. We use it to deal with the weak observability of discrete-time stochastic linear systems with Markovian jump and multiplicative noises in Section 3.2. 
A new necessary and sufficient condition for the weak observability will be given.

Notations. $R^{n}$ is $n$-dimensional real Euclidean space. $C^{n}$ is $n$ dimensional complex space. $A^{T},|A|$, and $\operatorname{tr}(A)$, respectively, denote the transpose, determinant, and trace of $A . A>$ $(\geq) 0$ means that $A$ is a symmetric positive (semipositive) definite matrix. $\mathbf{E}(\cdot)$ denotes the mathematical expectation of a random variable. $Z^{+} \triangleq\{0,1,2, \ldots\} . S \triangleq\{1,2, \ldots, s\}$, where $s$ is a positive integer. $R^{n \times m}$ denotes $n \times m$ real matrix space with the inner product $\langle A, B\rangle \triangleq \operatorname{tr}\left(A^{T} B\right) \cdot R_{s}^{n \times m} \triangleq$ $\left\{\left(U_{1}, \ldots, U_{s}\right) \mid U_{i} \in R^{n \times m}, i \in S\right\}$ with the inner product $\langle U, V\rangle \triangleq \sum_{i=1}^{s} \operatorname{tr}\left(U_{i}^{T} V_{i}\right) .1_{A}(x) \triangleq \begin{cases}1 & x \in \mathrm{A}, \\ 0 & x \notin A .\end{cases}$

\section{Transformation Matrix and Its Properties}

\subsection{Transformation Matrix between Two Vector Operators}

Definition 1 (see [1]). $L_{1}: C^{n \times m} \rightarrow C^{n m \times 1}$ is called a vector operator by column, if $L_{1}(X)=\left(x_{11}, x_{21}, \ldots, x_{n 1}, x_{12}\right.$, $\left.x_{22}, \ldots, x_{n 2}, \ldots, x_{1 m}, x_{2 m}, \ldots, x_{n m}\right)^{T}$ for any $X=\left(x_{i j}\right)_{n \times m}$.

Definition 2. $L_{2}: W^{n \times n} \rightarrow C^{(n(n+1) / 2) \times 1}$ is called a half vector operator by column, if $L_{2}(X)=\left(x_{11}, x_{21}, \ldots, x_{n 1}\right.$, $\left.x_{22}, \ldots, x_{n 2}, \ldots, x_{n n}\right)^{T}$ for any $X=\left(x_{i j}\right)_{n \times n}$, where $W^{n \times n} \triangleq$ $\left\{X \mid X^{T}=X \in C^{n \times n}\right\}$.

Definition 3 (see [1]). For matrices $A=\left(a_{i j}\right)_{n \times m}$ and $B=$ $\left(b_{i j}\right)_{s \times t}$, one calls matrix $A \otimes B=\left(a_{i j} B\right)_{n \times m}$ the Kronecker product of matrices $A$ and $B$.

It is easy to find that $L_{1}$ and $L_{2}$ have many similar properties, such as the following:

(a) $L_{1}$ and $L_{2}$ are all linear operators,

(b) $X=Y \Leftrightarrow L_{1}(X)=L_{1}(Y)$,

(c) $X=Y \Leftrightarrow L_{2}(X)=L_{2}(Y)$, where $X, Y \in W^{n \times n}$.

For convenience, we will adopt the following notations.

$E_{n, i, j}$ denotes a $n \times n$ matrix; its $(i, j)$-element is 1 , and it is zero elsewhere.

$H(n)$ denotes a $n^{2} \times(n(n+1) / 2)$ matrix. Its column vectors are denoted by $H^{11}, H^{21}, \ldots, H^{n 1}, H^{22}, \ldots, H^{n 2}, \ldots, H^{n n}$ from left to right and row vectors are denoted by $H_{11}, H_{21}, \ldots, H_{n 1}, \ldots, H_{1 n}, H_{2 n}, \ldots, H_{n n}$ from top to bottom, where

$$
H^{i j} \triangleq \begin{cases}L_{1}\left(E_{n, i, j}\right) & i=j, \\ L_{1}\left(E_{n, i, j}+E_{n, j, i}\right) & i>j .\end{cases}
$$

It is easy to prove

$$
H_{i j}= \begin{cases}L_{2}^{T}\left(E_{n, j, i}\right) & i<j \\ L_{2}^{T}\left(E_{n, i, j}\right) & i \geq j .\end{cases}
$$

We will denote $H=H_{n} \triangleq H(n)$.

$H^{-}(n)$ denotes a $(n(n+1) / 2) \times n^{2}$ matrix. Its column vectors are denoted by $H^{-11}, H^{-21}, \ldots, H^{-n 1}, \ldots$,
$H^{-1 n}, H^{-2 n}, \ldots, H^{-n n}$ from left to right and row vectors are denoted by $H_{11}^{-}, H_{21}^{-}, \ldots, H_{n 1}^{-}, H_{22}^{-}, \ldots, H_{n 2}^{-}, \ldots, H_{n n}^{-}$from top to bottom, where $H_{i j}^{-} \triangleq L_{1}^{T}\left(E_{n, i, j}\right)$. We will denote $H^{-}=H_{n}^{-} \triangleq H^{-}(n)$.

For example,

$$
\begin{array}{cc}
H(1)=[1], & H^{-}(1)=[1], \\
H(2)=\left[\begin{array}{lll}
1 & 0 & 0 \\
0 & 1 & 0 \\
0 & 1 & 0 \\
0 & 0 & 1
\end{array}\right], & H^{-}(2)=\left[\begin{array}{llll}
1 & 0 & 0 & 0 \\
0 & 1 & 0 & 0 \\
0 & 0 & 0 & 1
\end{array}\right],
\end{array}
$$

and so forth.

Considering Property 3, we generally call $\mathrm{H}$ and $\mathrm{H}^{-}$ transformation matrices between $L_{1}$ and $L_{2}$.

\subsection{Properties of Transformation Matrix}

Property 1. Consider

$$
H_{n}^{-} H_{n}=I_{n(n+1) / 2} \text {. }
$$

Proof. For $k=l$,

$$
H_{i j}^{-} H^{k l}=L_{1}^{T}\left(E_{n, i, j}\right) L_{1}\left(E_{n, k, k}\right)= \begin{cases}1 & i=j=k \\ 0 & \text { others. }\end{cases}
$$

For $k>l$,

$$
H_{i j}^{-} H^{k l}=L_{1}^{T}\left(E_{n, i, j}\right) L_{1}\left(E_{n, k, l}+E_{n, l, k}\right)= \begin{cases}1 & i=k, j=l \\ 0 & \text { others. }\end{cases}
$$

Thus, $H_{n}^{-} H_{n}=I_{n(n+1) / 2}$.

Remark 4. Generally $H_{n} H_{n}^{-} \neq I_{n^{2}}$, such as

$$
H_{2} H_{2}^{-}=\left[\begin{array}{llll}
1 & 0 & 0 & 0 \\
0 & 1 & 0 & 0 \\
0 & 1 & 0 & 0 \\
0 & 0 & 0 & 1
\end{array}\right] \text {. }
$$

Property 2. Consider

$$
H H^{-} H=H, H^{-} H H^{-}=H^{-},\left(H^{-} H\right)^{T}=H^{-} H .
$$

Remark 5. Generally $\left(H H^{-}\right)^{T} \neq H H^{-}$, such as $\mathrm{H}_{2} \mathrm{H}_{2}^{-}$which is not a symmetric matrix. This indicates that $H^{-}$is the $\{1,2,3\}-$ generalized inverse matrix of $H$, but not the $\{1,2,3,4\}$ generalized inverse matrix of $H$ (see [1]).

Property 3. If $X^{T}=X \triangleq\left(x_{i j}\right)_{n \times n}$, then $H L_{2}(X)=L_{1}(X)$, $H^{-} L_{1}(X)=L_{2}(X)$. 
Proof. Consider

$$
\begin{aligned}
\because H_{i j} L_{2}(X) & =L_{2}^{T}\left(E_{n, i, j}\right) L_{2}(X)=x_{i j} \quad(i \geq j), \\
H_{i j} L_{2}(X) & =L_{2}^{T}\left(E_{n, j, i}\right) L_{2}(X)=x_{j i}=x_{i j} \quad(i<j), \\
\therefore H L_{2}(X) & =L_{1}(X), \\
L_{2}(X) & =H^{-} L_{1}(X)(\text { by Property } 1) .
\end{aligned}
$$

Property 4. For matrices $A_{n \times n}, B_{r \times n}$ and $X_{n \times n}^{T}=X$, we have the following:

(a) $L_{1}\left(A X+X A^{T}\right)=(I \otimes A+A \otimes I) L_{1}(X)$,

(b) $L_{1}\left(B X B^{T}\right)=(B \otimes B) L_{1}(X)$,

(c) $L_{2}\left(A X+X A^{T}\right)=H^{-}(I \otimes A+A \otimes I) H L_{2}(X)$,

(d) $L_{2}\left(B X B^{T}\right)=H^{-}(B \otimes B) H L_{2}(X)$.

Proof. We can get (a) and (b) in [1], so we only need to prove (c) and (d). fore

Obviously, $A X+X A^{T}$ and $B X B^{T}$ are symmetrical. There-

$$
\begin{aligned}
& L_{2}\left(A X+X A^{T}\right)=H^{-} L_{1}\left(A X+X A^{T}\right) \\
& =H^{-}(I \otimes A+A \otimes I) L_{1}(X) \\
& =H^{-}(I \otimes A+A \otimes I) H L_{2}(X), \\
& L_{2}\left(B X B^{T}\right)=H^{-} L_{1}\left(B X B^{T}\right)=H^{-}(B \otimes B) H L_{2}(X) .
\end{aligned}
$$

Remark 6. If $L_{1}$ and $L_{2}$ are defined by row, that is,

$$
\begin{aligned}
L_{1}(X)= & \left(x_{11}, x_{12}, \ldots, x_{1 m}, x_{21}, x_{22}, \ldots,\right. \\
& \left.x_{2 m}, \ldots, x_{n 1}, x_{n 2}, \ldots, x_{n m}\right)^{T} \text { for } X \in C^{n \times m}, \\
L_{2}(X)= & \left(x_{11}, x_{12}, \ldots, x_{1 n}, x_{22}, \ldots,\right. \\
& \left.x_{2 n}, \ldots, x_{n n}\right)^{T} \text { for } X \in W^{n \times n},
\end{aligned}
$$

we find that Properties 3 and 4 are still true.

Property 5. For all $A \in C^{n \times n}$, then

(a) $\sigma(A \otimes A)=\sigma\left(H^{-}(A \otimes A) H\right)=\sigma\left(H^{-}\left(A^{T} \otimes A^{T}\right) H\right)$,

(b) $\sigma(A \otimes I+I \otimes A)=\sigma\left(H^{-}(A \otimes I+I \otimes A) H\right)=\sigma\left(H^{-}\left(A^{T} \otimes\right.\right.$ $\left.\left.I+I \otimes A^{T}\right) H\right)$

where $\sigma(\cdot)$ denotes the set of all eigenvalues of matrix.
Proof. (a) Let

$$
\begin{aligned}
\sigma_{1}(A) & \triangleq\left\{\lambda \in C \mid A X A^{T}=\lambda X, X \neq 0, X \in C^{n \times n}\right\}, \\
\sigma_{2}(A) & \triangleq\left\{\lambda \in C \mid A X A^{T}=\lambda X, X^{T}=X \neq 0, X \in C^{n \times n}\right\}, \\
\because A X A^{T} & =\lambda X \Longleftrightarrow(A \otimes A) L_{1}(X)=\lambda L_{1}(X) \\
\therefore & \sigma_{1}(A)=\sigma(A \otimes A) .
\end{aligned}
$$

If $X^{T}=X$, then $A X A^{T}=\lambda X \Leftrightarrow H^{-}(A \otimes A) H L_{2}(X)=$ $\lambda L_{2}(X)$. Thus, $\sigma_{2}(A)=\sigma\left(H^{-}(A \otimes A) H\right)$.

Next we prove $\sigma_{1}(A)=\sigma_{2}(A)$. Obviously $\sigma_{2}(A) \subseteq \sigma_{1}(A)$, so we only need to prove $\sigma_{1}(A) \subseteq \sigma_{2}(A)$.

For any $\lambda \in \sigma_{1}(A), \exists \hat{\lambda}, \widetilde{\lambda} \in \sigma(A)$ such that $\lambda=\hat{\lambda} \widetilde{\lambda}, \hat{\lambda} x=$ $A x$ with $0 \neq x \in C^{n}, \tilde{\lambda} y=A y$ with $0 \neq y \in C^{n}$ (see [1]). Let $X \triangleq x y^{T}, Z \triangleq X+X^{T}$; then

$$
\begin{aligned}
\lambda Z & =\hat{\lambda} \tilde{\lambda} x y^{T}+\hat{\lambda} \tilde{\lambda} y x^{T} \\
& =A x y^{T} A^{T}+A y x^{T} A^{T}=A Z A^{T} .
\end{aligned}
$$

If $Z \neq 0$, then $\lambda \in \sigma_{2}(A)$. Actually we always have $Z \neq 0$.

Suppose that $Z=0$; then $z_{i i}=0$ for $i=1,2, \ldots, n$ (i.e., $x_{i} y_{i}=0$ for $\left.i=1,2, \ldots, n\right)$. Without loss of generality, let $x_{i} \neq 0, y_{j} \neq 0$; then $y_{i}=0, x_{j}=0, z_{i j}=x_{i} y_{j}+y_{i} x_{j}=x_{i} y_{j} \neq 0$. This contradicts $Z=0$. Then $Z \neq 0$.

Thus, $\sigma(A \otimes A)=\sigma_{1}(A)=\sigma_{2}(A)=\sigma\left(H^{-}(A \otimes A) H\right)$. And because $\sigma(A \otimes A)=\sigma_{1}\left(A^{T} \otimes A^{T}\right)=\sigma\left(H^{-}\left(A^{T} \otimes A^{T}\right) H\right)$, therefore $\sigma(A \otimes A)=\sigma\left(H^{-}(A \otimes A) H\right)=\sigma\left(H^{-}\left(A^{T} \otimes A^{T}\right) H\right)$. (b) Let

$$
\begin{gathered}
\sigma_{3}(A) \triangleq\left\{\lambda \in C \mid A X+X A^{T}=\lambda X, X \neq 0, X \in C^{n \times n}\right\}, \\
\sigma_{4}(A) \triangleq\left\{\lambda \in C \mid A X+X A^{T}=\lambda X, X^{T}=X \neq 0, X \in C^{n \times n}\right\}, \\
\because A X+X A^{T}=\lambda X \Longleftrightarrow(A \otimes I+I \otimes A) L_{1}(X)=\lambda L_{1}(X) \\
\therefore \sigma_{3}(A)=\sigma(A \otimes I+I \otimes A) .
\end{gathered}
$$

If $X^{T}=X$, then $A X+X A^{T}=\lambda X \Leftrightarrow H^{-}(A \otimes I+I \otimes$ A) $H L_{2}(X)=\lambda L_{2}(X)$.

Thus, $\sigma_{4}(A)=\sigma\left(H^{-}(A \otimes I+I \otimes A) H\right)$.

Next we prove $\sigma_{3}(A)=\sigma_{4}(A)$. Obviously $\sigma_{4}(A) \subseteq \sigma_{3}(A)$, so we only need to prove $\sigma_{3}(A) \subseteq \sigma_{4}(A)$.

For any $\lambda \in \sigma_{3}(A), \exists \widehat{\lambda}, \widetilde{\lambda} \in \sigma(A)$ such that $\lambda=\hat{\lambda}+\tilde{\lambda}$, $\hat{\lambda} x=A x$ with $0 \neq x \in C^{n}, \tilde{\lambda} y=A y$ with $0 \neq y \in C^{n}$ (see [1]). Let $X \triangleq x y^{T}, Z \triangleq X+X^{T}$; then

$$
\begin{aligned}
\lambda Z & =\hat{\lambda} x y^{T}+\tilde{\lambda} x y^{T}+\hat{\lambda} y x^{T}+\tilde{\lambda} y x^{T} \\
& =A x y^{T}+x y^{T} A^{T}+y x^{T} A^{T}+A y x^{T} \\
& =A Z+Z A^{T} .
\end{aligned}
$$

If $Z \neq 0$, then $\lambda \in \sigma_{4}(A)$. Actually, we always have $Z \neq 0$.

Suppose that $Z=0$; then $z_{i i}=0$ for $i=1,2, \ldots, n$ (i.e., $x_{i} y_{i}=0$ for $\left.i=1,2, \ldots, n\right)$. Without loss of generality, let 
$x_{i} \neq 0, y_{j} \neq 0$; then $y_{i}=0, x_{j}=0, z_{i j}=x_{i} y_{j}+y_{i} x_{j}=x_{i} y_{j} \neq 0$. This contradicts $Z=0$. Then $Z \neq 0$.

Thus, $\sigma(A \otimes I+I \otimes A)=\sigma_{3}(A)=\sigma_{4}(A)=\sigma\left(H^{-}(A \otimes I+\right.$ $I \otimes A) H)$.

And because $\sigma(A \otimes I+I \otimes A)=\sigma_{1}\left(A^{T} \otimes I+I \otimes A^{T}\right)=$ $\sigma\left(H^{-}\left(A^{T} \otimes I+I \otimes A^{T}\right) H\right)$, therefore $\sigma(A \otimes I+I \otimes A)=\sigma\left(H^{-}(A \otimes\right.$ $I+I \otimes A) H)=\sigma\left(H^{-}\left(A^{T} \otimes I+I \otimes A^{T}\right) H\right)$.

\section{Applications of Transformation Matrix}

3.1. Application 1: The Improvement of Qian Jiling's Formula. Qian Jiling's formula is an important and interesting formula to compute the Lyapunov functions [2]. However, the formula can be improved with the help of the above transformation matrices.

Theorem 7. Assume that the system $\dot{x}(t)=A x(t)$ is asymptotically stable (i.e., $A \in R^{n \times n}$ is a Hurwitz matrix); then one has the following:

(a) for any $D \in R^{n \times n}, A^{T} Y+Y A=D$, there exists a unique solution $Y \in R^{n \times n}$,

(b) for any $D>0$, there exists a unique quadratic Lyapunov function $V=x^{T} Y x$, such that $A^{T} Y+Y A=$ $-D$, and the expression is

$$
V=\frac{1}{|\Delta|}\left|\begin{array}{cc}
0 & X \\
L_{2}(D) & \Delta
\end{array}\right|,
$$

where

$$
\begin{aligned}
\Delta & =H^{-}\left(I \otimes A^{T}+A^{T} \otimes I\right) H, \\
X & =\left(X_{1}, X_{2}, \ldots, X_{n}\right), \\
X_{1} & =\left(x_{1}^{2}, 2 x_{1} x_{2}, \ldots, 2 x_{1} x_{n}\right), \\
X_{2} & =\left(x_{2}^{2}, 2 x_{2} x_{3}, \ldots, 2 x_{2} x_{n}\right), \\
& \vdots \\
X_{n} & =\left(x_{n}^{2}\right) .
\end{aligned}
$$

Proof. We can get (a) in [2], so we only need to prove (b).

Let $Y=\int_{0}^{\infty} e^{t A^{T}} D e^{t A} \mathrm{~d} t$. Because $D>0$ and $A$ is a Hurwitz matrix, so $Y>0$ and

$$
\begin{aligned}
A^{T} Y+Y A & =A^{T} \int_{0}^{\infty} e^{t A^{T}} D e^{t A} \mathrm{~d} t+\int_{0}^{\infty} e^{t A^{T}} D e^{t A} \mathrm{~d} t A \\
& =\int_{0}^{\infty} \mathrm{d}\left(e^{t A^{T}} D e^{t A}\right)=-D
\end{aligned}
$$

By (a), the quadratic Lyapunov function $V=x^{T} Y x$ which satisfies $A^{T} Y+Y A=-D$ is unique.
Next we prove that $V$ can be expressed as (15). Note that $A^{T} Y+Y A=-D$; then

$$
H^{-}\left(I \otimes A^{T}+A^{T} \otimes I\right) H L_{2}(Y)=-L_{2}(D) .
$$

By (a), we have $\left|I \otimes A^{T}+A^{T} \otimes I\right| \neq 0$.

By Property 5, we have $\left|H^{-}\left(I \otimes A^{T}+A^{T} \otimes I\right) H\right| \neq 0$.

By the Cramer rule, (18) has a unique solution and

$$
y_{i j}=\frac{\left|\Delta^{* i j}\right|}{|\Delta|} \quad(1 \leq j \leq i \leq n) \text {, }
$$

where we use $-L_{2}(D)$ to replace $\Delta$ 's $(i, j)$-column in $\Delta$ and denote this new matrix by $\Delta^{* i j}(\Delta$ 's column vectors are, respectively, called the $(1,1),(2,1), \ldots,(n, 1),(2,2), \ldots$, $(n, 2), \ldots,(n, n)$-column vector from left to right).

By expanding the determinant, we have

$$
\begin{aligned}
\frac{1}{|\Delta|}\left|\begin{array}{cc}
0 & X \\
L_{2}(D) & \Delta
\end{array}\right|= & \sum_{i=1}^{n} x_{i}^{2} \frac{\left|\Delta^{* i i}\right|}{|\Delta|} \\
& +\sum_{1 \leq j<i \leq n} 2 x_{j} x_{i} \frac{\left|\Delta^{* i j}\right|}{|\Delta|} \\
= & \sum_{i, j=1}^{n} x_{i} y_{i j} x_{j}=V .
\end{aligned}
$$

For the uniqueness of $V,(15)$ is the desired expression.

Remark 8. The dimensions of $\Delta$ and $\left[\begin{array}{cc}0 & X \\ L_{2}(D) & \Delta\end{array}\right]$ in (15) are significantly smaller than those in [2] due to the application of the transformation matrices $H$ and $H^{-}$.

3.2. Application 2: New Results for the Observability of Stochastic Linear Systems. This subsection considers the observability of discrete-time stochastic linear systems. A new necessary and sufficient condition for the weak observability will be given with the help of these transformation matrices.

3.2.1. Description of the Stochastic Linear Systems. This subsection considers the following discrete-time stochastic linear system with Markovian jump and multiplicative noises:

$$
\begin{gathered}
x(k+1)=A_{0, \theta(k)} x(k)+B_{\theta(k)} u(k) \\
+\sum_{j=1}^{N} A_{j, \theta(k)} x(k) w_{j}(k), \\
y(k)=C_{0, \theta(k)} x(k)+\sum_{j=1}^{N} C_{j, \theta(k)} x(k) w_{j}(k),
\end{gathered}
$$

for $k \in Z^{+}$, where $x(k) \in R^{n}, u(k) \in R^{m}$, and $y(k) \epsilon$ $R^{r}$, respectively, denote the system state, control input, and measured output. $A_{i j} \in R^{n \times n}, B_{j} \in R^{n \times m}$, and $C_{i j} \in R^{r \times n}$. $\left\{\theta(k) ; k \in Z^{+}\right\}$is a discrete-time homogeneous Markovian chain. Its state space is $S$, transition probability matrix is 
$P=\left(p_{i j}\right)_{s \times s}$, and initial distribution is $\mu_{0}=\left\{\mu_{0, i} \triangleq\right.$ $P(\theta(0)=i)\}_{i \in S} \cdot w_{1}(k), \ldots, w_{N}(k) \in R$ are wide sense stationary processes and independent of each other, such that

$$
\mathbf{E}\left(w_{i}(k)\right)=0, \quad \mathbf{E}\left(w_{i}(k) w_{j}(k)\right)=\delta_{i j} \triangleq \begin{cases}1 & i=j \\ 0 & i \neq j\end{cases}
$$

and all independent of $\left\{\theta(k) ; k \in Z^{+}\right\}$.

Let $\Theta \triangleq\{\mu \mid \mu$ be initial distribution of Markovian chain $\left.\left\{\theta(k) ; k \in Z^{+}\right\}\right\}$.

For the stochastic system (21), its solution and output processes with $x(0)=x_{0}$ and $\theta(0) \sim \mu_{0}$ are, respectively, denoted by $x\left(k, \omega, x_{0}, \mu_{0}\right)$ and $y\left(k, \omega, x_{0}, \mu_{0}\right)$. We will simply denote $x(k)=x\left(k, x_{0}, \mu_{0}\right) \triangleq x\left(k, \omega, x_{0}, \mu_{0}\right), y(k)=$ $y\left(k, x_{0}, \mu_{0}\right) \triangleq y\left(k, \omega, x_{0}, \mu_{0}\right)$.

If $u(k) \equiv 0$, the stochastic system (21) becomes

$$
\begin{gathered}
x(k+1)=A_{0, \theta(k)} x(k)+\sum_{j=1}^{N} A_{j, \theta(k)} x(k) w_{j}(k), \\
y(k)=C_{0, \theta(k)} x(k)+\sum_{j=1}^{N} C_{j, \theta(k)} x(k) w_{j}(k)
\end{gathered}
$$

for $k \in Z^{+}$

For convenience, we will use the following notations:

$$
\begin{gathered}
X_{i}(k) \triangleq \mathbf{E}\left(x(k) x^{T}(k) 1_{\theta(k)=i}\right), \\
\widehat{X}(k) \triangleq\left(X_{1}(k), \ldots, X_{s}(k)\right), \\
X(k) \triangleq \mathbf{E}\left(x(k) x^{T}(k)\right), \\
Y_{i}(k) \triangleq \mathbf{E}\left(y(k) y^{T}(k) 1_{\theta(k)=i}\right), \\
\widehat{Y}(k) \triangleq\left(Y_{1}(k), \ldots, Y_{s}(k)\right), \\
Y(k) \triangleq \mathbf{E}\left(y(k) y^{T}(k)\right), \\
\mathscr{W}_{0} \triangleq\left\{\widehat{X}(0) \mid x_{0} \in R^{n}, \mu_{0} \in \Theta, X_{i}(0) \triangleq x_{0} x_{0}^{T} \mu_{0, i}, i \in S\right\} .
\end{gathered}
$$

It is easy to get $X_{1}(k)+\cdots+X_{s}(k)=X(k), Y_{1}(k)+$ $\cdots+Y_{s}(k)=Y(k), \mathbf{E}|x(k)|^{2}=\mathbf{E}\left(x^{T}(k) x(k)\right)=\operatorname{tr}(X(k))=$ $\sum_{i=1}^{s} \operatorname{tr}\left(X_{i}(k)\right)=\langle\widehat{X}(k), \widehat{I}\rangle$, where $\widehat{I} \triangleq[I, \ldots, I] \in R_{s}^{n \times n}$. Specifically $\mathbf{E}|x(0)|^{2}=\left|x_{0}\right|^{2}$.

For the system (23), we define several operators in $R_{s}^{n \times n}$ as follows:

$$
\begin{gathered}
\mathscr{E}_{i}(U)=\sum_{j=1}^{s} p_{i j} U_{j}, \\
\mathscr{F}_{i}(U)=\sum_{l=0}^{N} A_{l i}^{T} \mathscr{E}_{i}(U) A_{l i}=\sum_{l=0}^{N} \sum_{j=1}^{s} p_{i j} A_{l i}^{T} U_{j} A_{l i},
\end{gathered}
$$

$$
\begin{gathered}
\mathscr{F}_{i}^{*}(U)=\sum_{l=0}^{N} \sum_{j=1}^{s} p_{j i} A_{l j} U_{j} A_{l j}^{T}, \\
\mathscr{E}(U)=\left(\mathscr{E}_{1}(U), \ldots, \mathscr{C}_{s}(U)\right), \\
\mathscr{F}(U)=\left(\mathscr{F}_{1}(U), \ldots, \mathscr{F}_{s}(U)\right), \\
\mathscr{F}^{*}(U)=\left(\mathscr{F}_{1}^{*}(U), \ldots, \mathscr{F}_{s}^{*}(U)\right),
\end{gathered}
$$

$$
\begin{aligned}
& \mathscr{F}^{k}(U) \\
& \quad=\mathscr{F}\left(\mathscr{F}^{k-1}(U)\right) \quad(k=1,2, \ldots), \text { where } \mathscr{F}^{0}(U)=U, \\
& \mathscr{F}^{* k}(U) \\
& =\mathscr{F}^{*}\left(\mathscr{F}^{* k-1}(U)\right) \quad(k=1,2, \ldots), \text { where } \mathscr{F}^{* 0}(U)=U .
\end{aligned}
$$

$\mathscr{F}^{*}$ and $\mathscr{F}$ are called dual operators for each other (see Lemma 15).

Also, we define the operator $\mathscr{O}_{i}(l)=C_{i}+\mathscr{F}_{i}(\mathcal{O}(l-$ 1)) for $i \in S, l=1,2, \ldots$, where

$$
\begin{gathered}
C_{i}=\sum_{l=0}^{N} C_{l i}^{T} C_{l i}, \\
\mathcal{O}(0)=0,
\end{gathered}
$$

$$
\mathcal{O}(l)=\left(\mathscr{O}_{1}(l), \ldots, \mathcal{O}_{s}(l)\right) .
$$

Then

$$
\begin{array}{r}
\mathcal{O}(l)=\widehat{C}+\mathscr{F}(\mathcal{O}(l-1)) \quad\left(\widehat{C} \triangleq\left(C_{1}, \ldots, C_{s}\right)\right) \\
=\widehat{C}+\mathscr{F}(\widehat{C})+\mathscr{F}^{2}(\widehat{C})+\cdots+\mathscr{F}^{l-1}(\widehat{C}) \\
l=1,2, \ldots
\end{array}
$$

3.2.2. Some Preliminaries and Auxiliary Results. Let

$$
\begin{aligned}
& W_{i}^{l}(\widehat{X}(t)) \triangleq \sum_{k=0}^{l-1}\left\langle X_{i}(t+k), C_{i}\right\rangle \\
& \quad \text { for } t \in Z^{+}, i \in S, l=1,2, \ldots, \\
& W^{l}(\widehat{X}(t)) \triangleq \sum_{k=0}^{l-1}\langle\widehat{X}(t+k), \widehat{C}\rangle=\sum_{i=1}^{s} W_{i}^{l}(\widehat{X}(t))
\end{aligned}
$$

for $t \in Z^{+}, l=1,2, \ldots$

Lemma 9. For the system (23), one has $\mathscr{O}_{i}(l) \geq \mathcal{O}_{i}(l-1)$ for all $i \in S, l=1,2, \ldots$.

Proof. Firstly, it is easy to get $\mathcal{O}_{i}(1)=C_{i}=\sum_{l=0}^{N} C_{l i}^{T} C_{l i} \geq 0=$ $\mathcal{O}_{i}(0)$ for all $i \in S$. Secondly, we assume that $\mathcal{O}_{i}(l) \geq \mathcal{O}_{i}(l-1)$ for 
all $i \in S, l=1,2, \ldots, m$. Then, for $l=m+1$, for all $i \in S$, we have

$$
\begin{aligned}
\mathcal{O}_{i} & (m+1)-\mathcal{O}_{i}(m) \\
& =\mathscr{F}_{i}(\mathcal{O}(m))-\mathscr{F}_{i}(\mathcal{O}(m-1)) \\
& =\sum_{l=0}^{N} \sum_{j=1}^{s} p_{i j} A_{l i}^{T}\left(\mathcal{O}_{j}(m)-\mathcal{O}_{j}(m-1)\right) A_{l i} \\
& \geq 0 .
\end{aligned}
$$

By mathematical induction, it is right.

Lemma 10. For the system (23), one has $\mathscr{F}^{l+1}(\mathcal{O}(m-1))=$ $\mathscr{F}^{l}(\mathcal{O}(m))-\mathscr{F}^{l}(\widehat{C})$ for $m=1,2, \ldots, l=0,1, \ldots$

Proof. It is easy to get the result by mathematical induction, so we will omit the details.

Lemma 11. For the system (23), for all $x_{0} \in R^{n}$, for all $\mu_{0} \in \Theta$ (i.e., for all $\widehat{X}(0)=\widehat{X}_{0} \in \mathscr{W}_{0}$ ), one has

$$
\begin{array}{cc}
\widehat{X}(k+1)=\mathscr{F}^{*}(\widehat{X}(k)) & \text { for } k \in Z^{+}, \\
\widehat{X}(k+t) & =\mathscr{F}^{* t}(\widehat{X}(k)) \quad \text { for } k, t \in Z^{+} .
\end{array}
$$

Proof. For all $i \in S$, we have

$$
\begin{aligned}
& \mathscr{F}_{i}^{*}(\widehat{X}(k))=\sum_{l=0}^{N} \sum_{j=1}^{s} p_{j i} A_{l j} \mathbf{E}\left(x(k) x^{T}(k) 1_{\theta(k)=j}\right) A_{l j}^{T} \\
& =\sum_{l=0}^{N} \sum_{j=1}^{s} p_{j i} \mathrm{E}\left(A_{l, \theta(k)} x(k) x^{T}(k) A_{l, \theta(k)}^{T} 1_{\theta(k)=j}\right) \\
& =\sum_{l=0}^{N} \mathbf{E}\left(A_{l, \theta(k)} x(k) x^{T}(k) A_{l, \theta(k)}^{T} 1_{\theta(k+1)=i}\right) \\
& =\mathbf{E}\left\{\left[A_{0, \theta(k)} x(k)+\sum_{j=1}^{N} A_{j, \theta(k)} x(k) w_{j}(k)\right]\right. \\
& \times\left[A_{0, \theta(k)} x(k)\right. \\
& \left.\left.+\sum_{j=1}^{N} A_{j, \theta(k)} x(k) w_{j}(k)\right]^{T} 1_{\theta(k+1)=i}\right\} \\
& =\mathbf{E}\left\{x(k+1) x^{T}(k+1) 1_{\theta(k+1)=i}\right\} \\
& =X_{i}(k+1) \quad k \in Z^{+} \text {. }
\end{aligned}
$$

then $\widehat{X}(k+1)=\mathscr{F}^{*}(\widehat{X}(k))$ for $k \in Z^{+}$. $Z^{+}$.
Lemma 12. For the system (23), for all $x_{0} \in R^{n}$, for all $\mu_{0} \in \Theta$, one has

$$
\begin{aligned}
& W_{i}^{l}(\widehat{X}(t))=\sum_{k=0}^{l-1} \mathbf{E}\left[y^{T}(t+k) y(t+k) 1_{\theta(t+k)=i}\right] \\
& \text { for } t \in Z^{+}, i \in S, l=1,2, \ldots, \\
& W^{l}(\widehat{X}(t))=\sum_{k=0}^{l-1} \mathbf{E}\left[y^{T}(t+k) y(t+k)\right]
\end{aligned}
$$

$$
\text { for } t \in Z^{+}, l=1,2, \ldots \text {. }
$$

Proof. Because

$$
\begin{aligned}
Y_{i}(k)=\mathbf{E}\{ & {\left[C_{0, \theta(k)} x(k)+\sum_{j=1}^{N} C_{j, \theta(k)} x(k) w_{j}(k)\right] } \\
& \left.\times\left[C_{0, \theta(k)} x(k)+\sum_{j=1}^{N} C_{j, \theta(k)} x(k) w_{j}(k)\right]^{T} 1_{\theta(k)=i}\right\} \\
= & \sum_{l=0}^{N} C_{l, i} X_{i}(k) C_{l, i}^{T},
\end{aligned}
$$

therefore

$$
\begin{aligned}
\left\langle X_{i}(k), C_{i}\right\rangle & =\operatorname{tr}\left[X_{i}(k)\left(\sum_{l=0}^{N} C_{l i}^{T} C_{l i}\right)\right]=\operatorname{tr}\left(Y_{i}(k)\right), \\
W_{i}^{l}(\widehat{X}(t)) & =\sum_{k=0}^{l-1}\left\langle X_{i}(t+k), C_{i}\right\rangle=\sum_{k=0}^{l-1} \operatorname{tr}\left(Y_{i}(t+k)\right) \\
& =\sum_{k=0}^{l-1} \mathbf{E}\left[y^{T}(t+k) y(t+k) 1_{\theta(t+k)=i}\right], \\
\langle\widehat{X}(k), & \widehat{C}\rangle=\sum_{i=1}^{s} \operatorname{tr}\left(X_{i}(k) C_{i}\right)=\operatorname{tr}(Y(k)), \\
W^{l}(\widehat{X}(t))= & \sum_{k=0}^{l-1}\langle\widehat{X}(t+k), \widehat{C}\rangle=\sum_{k=0}^{l-1} \operatorname{tr}(Y(t+k)) \\
& =\sum_{k=0}^{l-1} \mathbf{E}\left[y^{T}(t+k) y(t+k)\right] .
\end{aligned}
$$

Remark 13. There is a physical interpretation. $W^{l}(\widehat{X}(t))$ is the accumulated energy of the output process $y(k)$ on the interval $t \leq k \leq t+l-1$. The $i$ th modal is $W_{i}^{l}(\widehat{X}(t))$.

Lemma 14. For the system (23), for all $x_{0} \in R^{n}$, for all $\mu_{0} \in \Theta$, $t \in Z^{+}$, one has $W^{l}(\widehat{X}(t))=\langle\widehat{X}(t), \mathcal{O}(l)\rangle$ for $l=1,2, \ldots$.

Proof. It is easy to get the result by mathematical induction, so we will omit the details. 
Lemma 15. For the system (23), for all $x_{0} \in R^{n}$, for all $\mu_{0} \in \Theta$, $m \in Z^{+}, t \in Z^{+}$, one has

$$
\left\langle\widehat{X}(t), \mathscr{F}^{l}(\mathscr{O}(m))\right\rangle=\left\langle\mathscr{F}^{* l}(\widehat{X}(t)), \mathcal{O}(m)\right\rangle \quad l \in Z^{+} .
$$

Proof. Firstly, it is easy to get

$$
\begin{aligned}
\left\langle\widehat{X}(t), \mathscr{F}^{0}(\mathcal{O}(m))\right\rangle \\
=\langle\widehat{X}(t), \mathcal{O}(m)\rangle \\
=\left\langle\mathscr{F}^{* 0}(\widehat{X}(t)), \mathcal{O}(m)\right\rangle \quad \forall m, t \in Z^{+} .
\end{aligned}
$$

Secondly, we assume that $\left\langle\widehat{X}(t), \mathscr{F}^{l}(\mathcal{O}(m))\right\rangle=\left\langle\mathscr{F}^{* l}(\widehat{X}(t))\right.$, $\mathcal{O}(m)\rangle$ for all $m, t \in Z^{+}, l=1,2, \ldots, q$. Then, for $l=q+1$, for all $m, t \in Z^{+}$, we have

$$
\begin{aligned}
\left\langle\widehat{X}(t), \mathscr{F}^{q+1}(\mathcal{O}(m))\right\rangle= & \left\langle\widehat{X}(t), \mathscr{F}^{q}(\mathcal{O}(m+1))\right\rangle \\
& -\left\langle\widehat{X}(t), \mathscr{F}^{q}(\widehat{C})\right\rangle \\
= & \left\langle\mathscr{F}^{* q}(\widehat{X}(t)), \mathcal{O}(m+1)\right\rangle \\
& -\left\langle\mathscr{F}^{* q}(\widehat{X}(t)), \widehat{C}\right\rangle \\
= & \langle\widehat{X}(t+q), \mathcal{O}(m+1)\rangle \\
& -\langle\widehat{X}(t+q), \widehat{C}\rangle \\
= & W^{m+1}(\widehat{X}(t+q))-\langle\widehat{X}(t+q), \widehat{C}\rangle \\
= & \sum_{k=0}^{m}\langle\widehat{X}(t+q+k), \widehat{C}\rangle \\
& -\langle\widehat{X}(t+q), \widehat{C}\rangle \\
= & \langle\widehat{X}(t+q+1), \mathcal{O}(m)\rangle \\
= & \left\langle\mathscr{F}^{* q+1}(\widehat{X}(t)), \mathscr{O}(m)\right\rangle .
\end{aligned}
$$

By mathematical induction, it is right.

Corollary 16. For the system (23), for all $x_{0} \in R^{n}$, for all $\mu_{0} \in$ $\Theta, t \in Z^{+}$, one has

$$
\left\langle\widehat{X}(t), \mathscr{F}^{l}(\widehat{C})\right\rangle=\mathbf{E}\left[y^{T}(t+l) y(t+l)\right] .
$$

Proof. Consider

$$
\begin{aligned}
\left\langle\widehat{X}(t), \mathscr{F}^{l}(\widehat{C})\right\rangle & =\left\langle\widehat{X}(t), \mathscr{F}^{l}(\mathcal{O}(1))\right\rangle \\
& =\left\langle\mathscr{F}^{* l}(\widehat{X}(t)), \mathcal{O}(1)\right\rangle \\
& =\langle\widehat{X}(t+l), \widehat{C}\rangle \\
& =\mathbf{E}\left[y^{T}(t+l) y(t+l)\right] .
\end{aligned}
$$

In particular, we have $\left\langle\widehat{X}(0), \mathscr{F}^{l}(\widehat{C})\right\rangle=\mathbf{E}\left[y^{T}(l) y(l)\right]$ when $t=0, m=1$.

Lemma 17 (see [12]). For any random variable $y \in R^{n}$, one has

$$
y=0 \quad \text { a.s. } \Longleftrightarrow \mathbf{E}\left(y y^{T}\right)=0 \Longleftrightarrow \mathbf{E}\left(y^{T} y\right)=0 .
$$

Lemma 18. For the system (23), for all $x_{0} \in R^{n}$, for all $\mu_{0} \in \Theta$, one has

$$
\lim _{k \rightarrow \infty} \mathbf{E}\left[x(k) x^{T}(k)\right]=0 \Longleftrightarrow \lim _{k \rightarrow \infty} \mathbf{E}\left[x^{T}(k) x(k)\right]=0 .
$$

Proof. This proof is omitted.

Lemma 19 (see [1]). If $0 \leq X \in R^{n \times n}, r \triangleq \operatorname{rank}(X)$, then there exist nonzero vectors $x_{1}, \ldots, x_{r} \in R^{n}$ such that $X=x_{1} x_{1}^{T}+$ $\cdots+x_{r} x_{r}^{T}$.

3.2.3. A Useful Formula. Define two operators $\mathscr{L}_{1}(U)=$ $\left[\begin{array}{c}L_{1}\left(U_{1}\right) \\ \vdots \\ L_{1}\left(U_{s}\right)\end{array}\right]$ for $U_{i} \in R^{n \times n}$ and $\mathscr{L}_{2}(U)=\left[\begin{array}{c}L_{2}\left(U_{1}\right) \\ \vdots \\ L_{2}\left(U_{s}\right)\end{array}\right]$ for $U_{i} \in\{X \mid$ $\left.X^{T}=X \in R^{n \times n}\right\}$ in $R_{s}^{n \times n}$.

For convenience, we naturally think $Y \in\left\{X \mid X^{T}=X \in\right.$ $\left.R^{n \times n}\right\}$ when we use $L_{2}(Y)$ in this subsection.

Theorem 20. For the system (23), for all $U, V \in R_{s}^{n \times n}$, one has

$$
\begin{aligned}
\mathscr{L}_{1}(\mathscr{F}(U)) & =\mathscr{A L}_{1}(U), \\
\mathscr{L}_{2}(\mathscr{F}(U)) & =\mathscr{B}_{2}(U), \\
\langle U, V\rangle & =\mathscr{L}_{1}^{T}(U) \mathscr{L}_{1}(V), \\
\langle U, V\rangle & =\mathscr{L}_{1}^{T}(U)(I \otimes H) \mathscr{L}_{2}(V),
\end{aligned}
$$

where

$$
\begin{gathered}
\mathscr{A} \triangleq \sum_{l=0}^{N} \operatorname{diag}\left(A_{l 1}^{T} \otimes A_{l 1}^{T}, \ldots, A_{l s}^{T} \otimes A_{l s}^{T}\right)(P \otimes I) \mathscr{L}_{1}(U), \\
\mathscr{B} \triangleq \sum_{l=0}^{N} \operatorname{diag}\left(H^{-}\left(A_{l 1}^{T} \otimes A_{l 1}^{T}\right) H, \ldots,\right. \\
\left.H^{-}\left(A_{l s}^{T} \otimes A_{l s}^{T}\right) H\right)(P \otimes I) \mathscr{L}_{2}(U) .
\end{gathered}
$$


Proof. When $U_{i}^{T}=U_{i}$ for $i \in S$, then

$$
\begin{aligned}
\mathscr{L}_{2}(\mathscr{F}(U)) & =\left[\begin{array}{c}
L_{2}\left(\sum_{l=0}^{N} \sum_{j=1}^{s} p_{1, j} A_{l 1}^{T} U_{j} A_{l 1}\right) \\
\vdots \\
L_{2}\left(\sum_{l=0}^{N} \sum_{j=1}^{s} p_{s j} A_{l s}^{T} U_{j} A_{l s}\right)
\end{array}\right] \\
& =\sum_{l=0}^{N}\left[\begin{array}{c}
H^{-}\left(A_{l 1}^{T} \otimes A_{l 1}^{T}\right) H \sum_{j=1}^{s} p_{1, j} L_{2}\left(U_{j}\right) \\
H^{-}\left(A_{l s}^{T} \otimes A_{l s}^{T}\right) H \sum_{j=1}^{s} p_{s j} L_{2}\left(U_{j}\right)
\end{array}\right] \\
& =\Sigma_{l=0}^{N} \operatorname{diag}\left(H^{-}\left(A_{l 1}^{T} \otimes A_{l 1}^{T}\right) H, \ldots,\right. \\
\left.H^{-}\left(A_{l s}^{T} \otimes A_{l s}^{T}\right) H\right)(P \otimes I) \mathscr{L}_{2}(U) & \\
= & \mathscr{B} \mathscr{L}_{2}(U) .
\end{aligned}
$$

By the same way, it is easy to get

$$
\begin{aligned}
& \mathscr{L}_{1}(\mathscr{F}(U))=\sum_{l=0}^{N} \operatorname{diag}\left(A_{l 1}^{T} \otimes A_{l 1}^{T}, \ldots,\right. \\
&\left.A_{l s}^{T} \otimes A_{l s}^{T}\right)(P \otimes I) \mathscr{L}_{1}(U)
\end{aligned}
$$

$=\mathscr{A} \mathscr{L}_{1}(U)$.

Consider

$$
\begin{aligned}
\langle U, V\rangle & =\sum_{i=1}^{s} \operatorname{tr}\left(U_{i}^{T} V_{i}\right)=\sum_{i=1}^{s} L_{1}^{T}\left(U_{i}\right) L_{1}\left(V_{i}\right) \\
& =\mathscr{L}_{1}^{T}(U) \mathscr{L}_{1}(V), \\
\langle U, V\rangle & =\sum_{i=1}^{s} L_{1}^{T}\left(U_{i}\right) L_{1}\left(V_{i}\right) \\
& =\sum_{i=1}^{s} L_{1}^{T}\left(U_{i}\right) H L_{2}\left(V_{i}\right) \\
& =\mathscr{L}_{1}^{T}(U)(I \otimes H) \mathscr{L}_{2}(V) .
\end{aligned}
$$

Let $d \triangleq s(n(n+1) / 2)$.

Remark 21. For the symmetric case, $\mathscr{L}_{2}(\cdot)$ is more suitable than $\mathscr{L}_{1}(\cdot)$ in applications (such as Lemma 22 and Theorem 26). Without the transformation matrices, it is very difficult to obtain these results. Thus, we say that the transformation matrices are an effective mathematical tool.
Lemma 22. For the system (23), for all $x_{0} \in R^{n}$, for all $\mu_{0} \in \Theta$, $t \in Z^{+}$, one has

$$
\begin{aligned}
W^{d}(\widehat{X}(t))=0 & \Longleftrightarrow W^{l}(\widehat{X}(k))=0 \quad(l \geq 1, k \geq t) \\
& \Longleftrightarrow E\left(y^{T}(k) y(k)\right)=0 \quad(k \geq t) .
\end{aligned}
$$

Proof. By Lemma 12, it is easy to get

$$
\begin{aligned}
& W^{l}(\widehat{X}(k))=0 \quad(l \geq 1, k \geq t) \\
& \Longleftrightarrow E\left(y^{T}(k) y(k)\right)=0 \quad(k \geq t) .
\end{aligned}
$$

Obviously, $W^{l}(\widehat{X}(k))=0(l \geq 1, k \geq t) \Rightarrow W^{d}(\widehat{X}(t))=0$.

We only need to prove $W^{d}(\widehat{X}(t))=0 \Rightarrow W^{l}(\widehat{X}(k))=$ $0(l \geq 1, k \geq t)$ :

$$
\begin{gathered}
\because \mathscr{L}_{2}(\mathscr{F}(U))=\mathscr{B}_{2}(U) \text { (by Theorem 20) } \\
\mathcal{O}(l)=\widehat{C}+\mathscr{F}(\mathcal{O}(l-1)) \\
\therefore \mathscr{L}_{2}(\mathcal{O}(l))=\mathscr{L}_{2}(\widehat{C})+\mathscr{B} \mathscr{L}_{2}(\mathcal{O}(l-1)) \\
=q+\mathscr{B}\left[q+\mathscr{B} \mathscr{L}_{2}(\mathcal{O}(l-2))\right]
\end{gathered}
$$

where $q \triangleq \mathscr{L}_{2}(\widehat{C})$

$$
=q+\mathscr{B} q+\cdots+\mathscr{B}^{l-1} q \quad(l=1,2, \ldots)
$$

$\because\left\langle\widehat{X}(t), \mathscr{F}^{l}(\widehat{C})\right\rangle$

$$
=\mathbf{E}\left[y^{T}(t+l) y(t+l)\right] \geq 0 \text { (by Corollary 16) }
$$

$\therefore\left\langle\widehat{X}(t), \mathscr{F}^{l}(\widehat{C})\right\rangle$

$$
\begin{aligned}
& =\mathscr{L}_{1}^{T}(\widehat{X}(t))(I \otimes H) \mathscr{L}_{2}\left(\mathscr{F}^{l}(\widehat{C})\right) \text { (by Theorem 20) } \\
& =\mathscr{L}_{1}^{T}(\widehat{X}(t))(I \otimes H) \mathscr{B} \mathscr{L}_{2}\left(\mathscr{F}^{l-1}(\widehat{C})\right) \\
& =\mathscr{L}_{1}^{T}(\widehat{X}(t))(I \otimes H) \mathscr{B}^{l} q \geq 0 \quad\left(l \in Z^{+}\right) .
\end{aligned}
$$

$$
\begin{aligned}
& \text { If } W^{d}(\widehat{X}(t))=0 \text {, then } \\
& \begin{aligned}
\langle\widehat{X}(t), \mathcal{O}(d)\rangle= & \mathscr{L}_{1}^{T}(\widehat{X}(t))(I \otimes H) \mathscr{L}_{2}(\mathcal{O}(d)) \\
= & \mathscr{L}_{1}^{T}(\widehat{X}(t))(I \otimes H) \\
& \times\left(q+\mathscr{B} q+\cdots+\mathscr{B}^{d-1} q\right) \\
= & 0 .
\end{aligned}
\end{aligned}
$$

Thus, $\mathscr{L}_{1}^{T}(\widehat{X}(t))(I \otimes H) \mathscr{B}^{k} q=0$ for $k=0,1, \ldots, d-1$.

For $\mathscr{B} \in R^{d \times d}$, there exist $a_{0}(l), a_{1}(l), \ldots, a_{d-1}(l) \in R$ by the Cayley-Hamilton theorem, such that

$$
\mathscr{B}^{l}=a_{0}(l) \mathscr{B}^{0}+a_{1}(l) \mathscr{B}^{1}+\cdots+a_{d-1}(l) \mathscr{B}^{d-1} \quad\left(l \in Z^{+}\right) .
$$


For $l \geq 1, k \geq t$, we have

$$
\begin{aligned}
W^{l}(\widehat{X}(k))= & \left\langle\widehat{X}(t), \mathscr{F}^{k-t}(\mathcal{O}(l))\right\rangle \\
= & \mathscr{L}_{1}^{T}(\widehat{X}(t))(I \otimes H) \mathscr{B}^{k-t} \mathscr{L}_{2}(\mathcal{O}(l)) \\
= & \mathscr{L}_{1}^{T}(\widehat{X}(t))(I \otimes H) \\
& \times\left(\mathscr{B}^{k-t}+\mathscr{B}^{k-t+1}+\cdots+\mathscr{B}^{k-t+l-1}\right) q \\
= & \mathscr{L}_{1}^{T}(\widehat{X}(t))(I \otimes H) \\
& \times\left[b_{0}(\nu) \mathscr{B}^{0}+b_{1}(\nu) \mathscr{B}^{1}\right. \\
& \left.+\cdots+b_{d-1}(\nu) \mathscr{B}^{d-1}\right] q \\
= & b_{0}(\nu) \mathscr{L}_{1}^{T}(\widehat{X}(t))(I \otimes H) \mathscr{B}^{0} q \\
& +b_{1}(\nu) \mathscr{L}_{1}^{T}(\widehat{X}(t))(I \otimes H) \mathscr{B}^{1} q \\
& +\cdots+b_{d-1}(\nu) \mathscr{L}_{1}^{T}(\widehat{X}(t))(I \otimes H) \mathscr{B}^{d-1} q \\
= & 0,
\end{aligned}
$$

where $b_{0}(\nu), b_{1}(\nu), \ldots, b_{d-1}(\nu) \in R$.

Corollary 23. For the system (23), for all $x_{0} \in R^{n}$, for all $\mu_{0} \in$ $\Theta$, one has $W^{d}\left(\widehat{X}_{0}\right)=0 \Leftrightarrow \mathbf{E}\left(y^{T}(k) y(k)\right)=0(k \geq 0)$.

\subsubsection{Main Results for the Weak Observability}

Definition 24. The stochastic linear system (23) is weak observable ( $W$-observable), if, for all $\mu_{0} \in \Theta$, one has

$$
y(k)=0 \quad \text { a.s., } \forall k \in Z^{+} \Longrightarrow x_{0}=0 .
$$

Remark 25. By Lemma 22, (53) is equivalent to

$$
W^{d}\left(\widehat{X}_{0}\right)=0 \Longrightarrow x_{0}=0
$$

Theorem 26. The system (23) is $W$-observable if and only if $\mathcal{O}_{i}(d)>0$ for all $i \in S$.

Proof. (Sufficiency) For all $\mu_{0} \in \Theta$, if $y(k)=0$ a.s. $\left(k \in Z^{+}\right)$, by Lemmas 14 and 22, we have

$$
\begin{aligned}
W^{d}\left(\widehat{X}_{0}\right) & =\left\langle\widehat{X}_{0}, \mathcal{O}(d)\right\rangle=\sum_{i=1}^{s} \operatorname{tr}\left(X_{i}(0) \mathcal{O}_{i}(d)\right) \\
& =\sum_{i=1}^{s} \mu_{0, i} x_{0}^{T} \mathcal{O}_{i}(d) x_{0}=0 .
\end{aligned}
$$

Because $\mathcal{O}_{i}(d)>0$ for all $i \in S$, so $x_{0}=0$.

That is, the system (23) is $W$-observable.

(Necessity) Suppose that the system (23) is $W$-observable; then, for all $\mu_{0} \in \Theta$, we have

$x_{0} \neq 0 \Longrightarrow \exists k_{0} \triangleq k_{0}\left(\mu_{0}, x_{0}\right) \quad$ such that $P\left(y\left(k_{0}\right)=0\right) \neq 1$.
By Corollary 23, if $W^{d}\left(\widehat{X}_{0}\right)=0$, then $\mathbf{E}\left(y^{T}(k) y(k)\right)=0$ for $k \in Z^{+}$. Thus we can consistently select $0 \leq k_{0} \leq d$ for above $x_{0}$, such that

$$
W^{d+1}\left(\widehat{X}_{0}\right)=\sum_{k=0}^{d} \mathbf{E}\left(y^{T}(k) y(k)\right) \geq \mathbf{E}\left(y^{T}\left(k_{0}\right) y\left(k_{0}\right)\right)>0 .
$$

Assume that there exists $i \in S$ such that $\mathcal{O}_{i}(d)$ is strictly semipositive definite (i.e., $\exists 0 \neq v \in R^{n}$ such that $v^{T} \mathscr{O}_{i}(d) v=$ $0)$. Select $\mu_{0} \in \Theta$ such that $\mu_{0, j}=0(j \neq i)$. Let $\widehat{X}_{0}=$ $\left(v v^{T} \mu_{o, 1}, \ldots, v v^{T} \mu_{o, s}\right)$; then

$$
W^{d}\left(\widehat{X}_{0}\right)=\left\langle\widehat{X}_{0}, \mathcal{O}(d)\right\rangle=\sum_{j=1}^{s} \mu_{0, j} v^{T} \mathcal{O}_{j}(d) v=0 .
$$

By Lemma $22, W^{d+1}\left(\widehat{X}_{0}\right)=0$. It contradicts $W^{d+1}\left(\widehat{X}_{0}\right)>0$. Thus, $\mathcal{O}_{i}(d)>0$ for $i \in S$.

Theorem 27. For the system (23), if $\exists k \in Z^{+}$such that $\mathscr{O}_{i}(k)>$ 0 for any $i \in S$, then $\mathcal{O}_{i}(k+1)>0$ for any $i \in S$.

Proof. Assume that there exists $i \in S$ such that $\mathcal{O}_{i}(k+1)$ is strictly semipositive definite (i.e., $\exists 0 \neq v \in R^{n}$ such that $\left.v^{T} \mathscr{O}_{i}(k+1) v=0\right)$. Select $\mu_{0} \in \Theta$ to satisfy $\mu_{0, j}=0(j \neq i)$. Let $\widehat{X}_{0}=\left(v v^{T} \mu_{o, 1}, \ldots, v v^{T} \mu_{o, s}\right)$; then

$$
W^{k+1}\left(\widehat{X}_{0}\right)=\left\langle\widehat{X}_{0}, \mathcal{O}(k+1)\right\rangle=\sum_{j=1}^{s} \mu_{0, j} v^{T} \mathcal{O}_{j}(k+1) v=0 .
$$

It contradicts $W^{k+1}\left(\widehat{X}_{0}\right) \geq W^{k}\left(\widehat{X}_{0}\right)=\left\langle\widehat{X}_{0}, \mathcal{O}(k)\right\rangle>0$. Thus $\mathcal{O}_{i}(k+1)>0$ for $i \in S$.

Theorem 28. For the system (23), the following two statements are equivalent:

(a) the system (23) is W-observable,

(b) $\exists N_{d} \geq 1, \gamma>0$, for all $x_{0} \in R^{n}$ and $\mu_{0} \in \Theta$; one has $W^{N_{d}}\left(\widehat{X}_{0}\right) \geq \gamma\left|x_{0}\right|^{2}$.

Proof. (a) $\Rightarrow$ (b) The system (23) is $W$-observable; then $\mathcal{O}_{i}(d)>0$ for $i \in S$. Select $N_{d}=d$; then

$$
\begin{aligned}
W^{d}\left(\widehat{X}_{0}\right)=\left\langle\widehat{X}_{0}, \mathcal{O}(d)\right\rangle & =\sum_{i=1}^{s} \mu_{0, i} x_{0}^{T} \mathcal{O}_{i}(d) x_{0} \\
& \geq \sum_{i=1}^{s}\left[\mu_{0, i} \lambda_{\min }\left(\mathcal{O}_{i}(d)\right)\right]\left|x_{0}\right|^{2} \\
& \geq \sum_{i=1}^{s}\left[\mu_{0, i} \gamma\right]\left|x_{0}\right|^{2}=\gamma\left|x_{0}\right|^{2}
\end{aligned}
$$

where $\gamma=\min \left\{\lambda_{\min }\left(\mathcal{O}_{1}(d)\right), \ldots, \lambda_{\min }\left(\mathscr{O}_{s}(d)\right)\right\}>0$.

(b) $\Rightarrow$ (a) For all $\mu_{0} \in \Theta$, if $y(k)=0$ a.s. $\left(k \in Z^{+}\right)$, then

$$
W^{l}\left(\widehat{X}_{0}\right)=\sum_{k=0}^{l-1} \mathbf{E}\left(y^{T}(k) y(k)\right)=0
$$


for $l=1,2, \ldots$ Because $0=W^{N_{d}}\left(\widehat{X}_{0}\right) \geq \gamma\left|x_{0}\right|^{2}$, so $x_{0}=0$. That is, the system (23) is $W$-observable.

By Theorem 28 and [6], it is easy to get the following theorem.

Theorem 29. For the system (23), the following two statements are equivalent:

(a) the system (23) is W-observable,

(b) $\exists k \in Z^{+}$, such that $\mathcal{O}_{i}(k)>0$ for all $i \in S$.

Remark 30. Based on the above theorems, we can determine that the $W$-observability in this paper is essentially consistent with [5]. We believe that Definition 24 in this paper is more aligned with the intuitive physical meanings. Furthermore, Theorem 26 gives a new necessary and sufficient condition for the $W$-observability.

If we do not consider the noises, that is, $A_{j, i}=C_{j, i}=0$ for $j=1, \ldots, N, i \in S$, (23) becomes the following Markovian jump linear system:

$$
\begin{gathered}
x(k+1)=A_{0, \theta(k)} x(k), \\
y(k)=C_{0, \theta(k)} x(k) .
\end{gathered}
$$

If the Markovian chain degenerates into an ordinary situation, that is, $S=\{1\}$, (23) becomes the following stochastic linear system:

$$
\begin{gathered}
x(k+1)=A_{0} x(k)+\sum_{j=1}^{N} A_{j} x(k) w_{j}(k), \\
y(k)=C_{0} x(k)+\sum_{j=1}^{N} C_{j} x(k) w_{j}(k),
\end{gathered}
$$

where $A_{j} \triangleq A_{j, 1}, C_{j} \triangleq C_{j, 1}$ for $j=0,1, \ldots, N$.

For the above two special situations, it is easy to get the following theorems.

Theorem 31. For the Markovian jump linear system (62), the following statements are equivalent:
(a) the Markovian jump linear system (62) is $W$ - observable,
(b) $\exists N_{d} \geq 1, \gamma>0$, for all $x_{0} \in R^{n}$ and $\mu_{0} \in \Theta$; one has $W^{N_{d}}\left(\widehat{X}_{0}\right) \geq \gamma\left|x_{0}\right|^{2}$,
(c) $\exists k \in Z^{+}$, such that $\mathcal{O}_{i}(k)>0$ for all $i \in S$,
(d) $\mathcal{O}_{i}(d)>0$ for all $i \in S$.

Theorem 32. For the stochastic linear system (63), the following statements are equivalent:

(a) the stochastic linear system (63) is W-observable,

(b) $\exists N_{d} \geq 1, \gamma>0$, for all $x_{0} \in R^{n}$; one has $W^{N_{d}}\left(x_{0} x_{0}^{T}\right) \geq$ $\gamma\left|x_{0}\right|^{2}$

(c) $\exists k \in Z^{+}$, such that $\mathcal{O}(k)>0$,

(d) $\mathcal{O}(d)>0$.
Remark 33. Theorems 31 and 32 have essential improvements compared with those theorems in [5-7] (because $d=s(n)+$ 1)/2) $\left.\leq s n^{2}\right)$. Furthermore, $\left\{\mathcal{O}_{i}(d)\right\}_{i \in S}$ are the characteristic matrices for the observability of discrete-time stochastic linear system (such as (23), (62), and (63)).

\section{Conclusions}

This paper studies a class of important transformation matrices between two different vector operators, gives some important properties of it, and demonstrates its applications with two examples. One is to improve Qian Jiling's formula. Another is to give a new necessary and sufficient condition for the $W$-observability of stochastic linear systems. We emphasize that the transformation matrices are not only used to study these two problems but also used to study other problems. Actually the transformation matrices are an effective mathematical tool in applications.

\section{Conflict of Interests}

The authors declare that there is no conflict of interests regarding the publication of this paper.

\section{Acknowledgments}

This work is supported by the National Natural Science Foundation under Grants 61273126, 61363002, and 61374104; the Natural Science Foundation of Guangdong Province under Grants 10251064101000008, S2012010009675; the Research Foundation for the Doctoral Program of Higher Education of China under Grant 20130172110027; and the Foundation of Key Laboratory of Autonomous Systems and Networked Control under Grant 2013A01.

\section{References}

[1] R. C. Shi and F. Wei, Matrix Analysis, Beijing Institute of Technology Press, Beijing, China, 3rd edition, 2010.

[2] J. L. Qian and X. X. Liao, "A new solution method for the matrix equation $A^{T} X+X B=C$ and its application," Journal of Central China Normal University, vol. 21, no. 2, pp. 159-165, 1987.

[3] D. Z. Zheng, Linear System Theory, Tsinghua University Press, Beijing, China, 2nd edition, 2002.

[4] B. D. O. Anderson and J. B. Moore, "Detectability and stabilizability of time-varying discrete-time linear systems," Journal on Control and Optimization, vol. 19, no. 1, pp. 20-32, 1981.

[5] E. F. Costa and J. B. R. do Val, "On the detectability and observability of discrete-time Markov jump linear systems," Systems \& Control Letters, vol. 44, no. 2, pp. 135-145, 2001.

[6] V. Dragan and T. Morozan, "Observability and detectability of a class of discrete-time stochastic linear systems," IMA Journal of Mathematical Control and Information, vol. 23, no. 3, pp. 371394, 2006.

[7] Z. Y. Li, Y. Wang, B. Zhou, and G. R. Duan, "Detectability and observability of discrete-time stochastic systems and their applications," Automatica, vol. 45, no. 5, pp. 1340-1346, 2009. 
[8] W. Zhang and B.-S. Chen, "On stabilizability and exact observability of stochastic systems with their applications," Automatica, vol. 40, no. 1, pp. 87-94, 2004.

[9] T. Damm, "On detectability of stochastic systems," Automatica, vol. 43, no. 5, pp. 928-933, 2007.

[10] W. Zhang, H. Zhang, and B.-S. Chen, "Generalized Lyapunov equation approach to state-dependent stochastic stabilization/detectability criterion," IEEE Transactions on Automatic Control, vol. 53, no. 7, pp. 1630-1642, 2008.

[11] J. W. Lee and P. P. Khargonekar, "Detectability and stabilizability of discrete-time switched linear systems," IEEE Transactions on Automatic Control, vol. 54, no. 3, pp. 424-437, 2009.

[12] S. S. Mao, Y. M. Cheng, and X. L. Pu, Probability Theory and Mathematical Statistics, Higher education press, Beijing, China, 2004. 


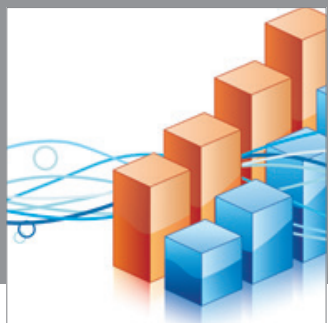

Advances in

Operations Research

mansans

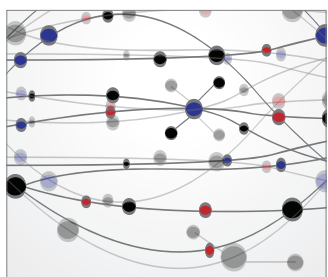

The Scientific World Journal
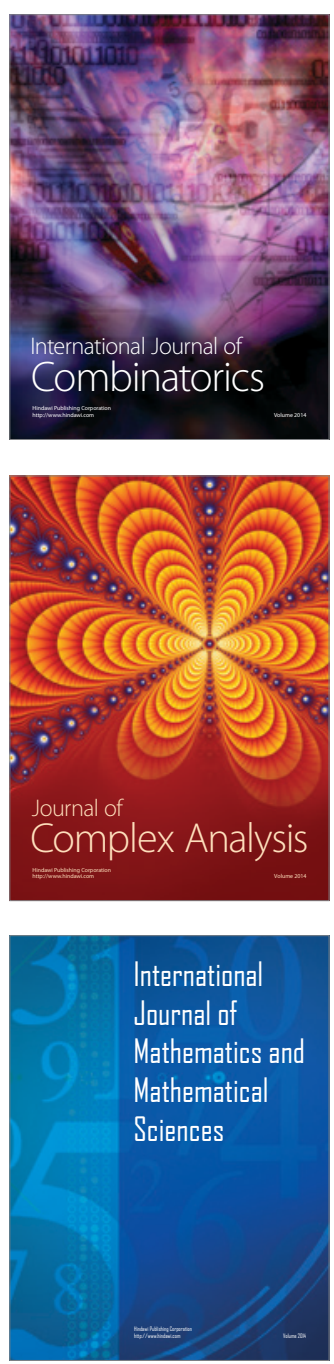
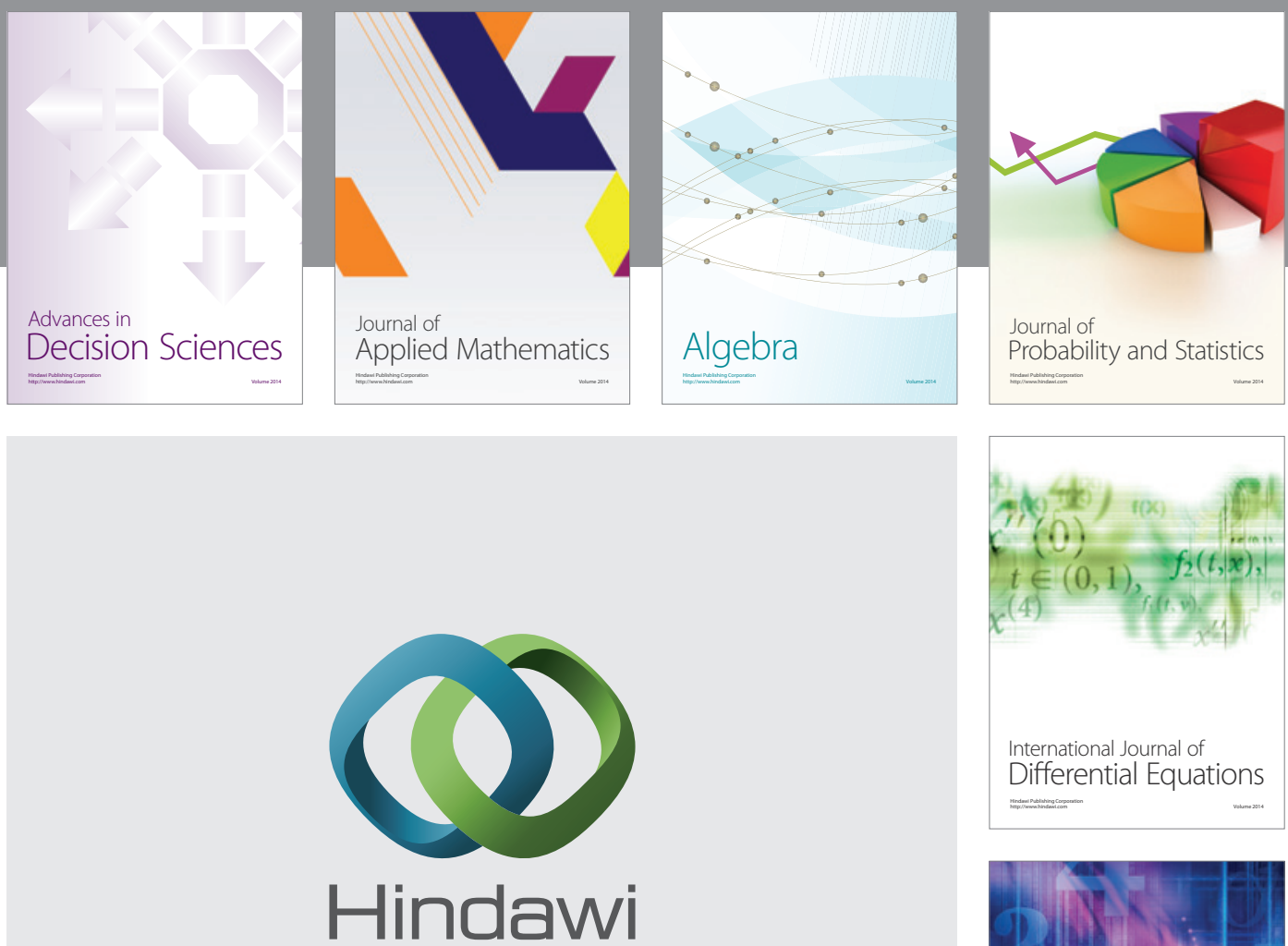

Submit your manuscripts at http://www.hindawi.com
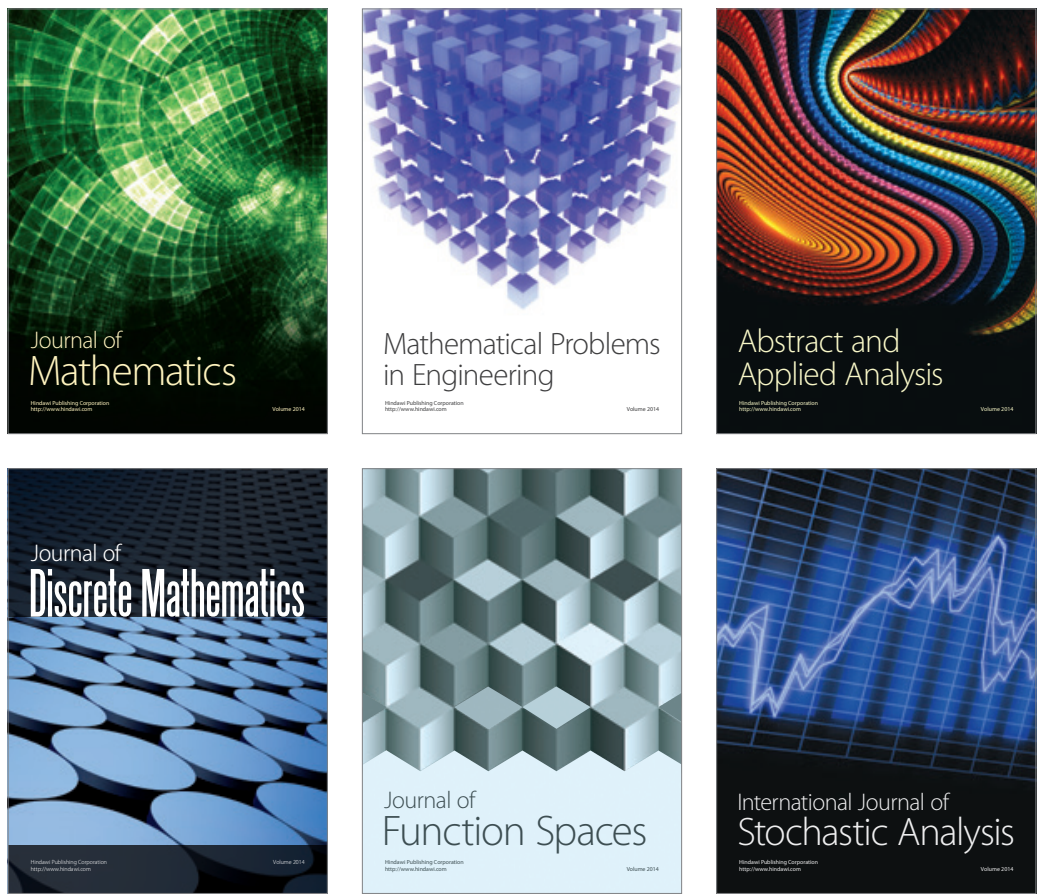

Journal of

Function Spaces

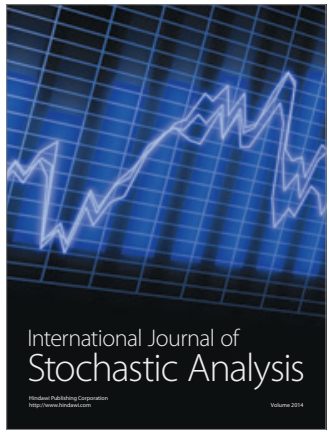

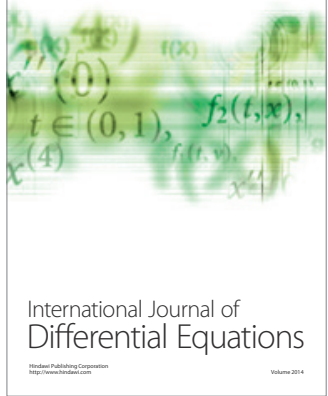
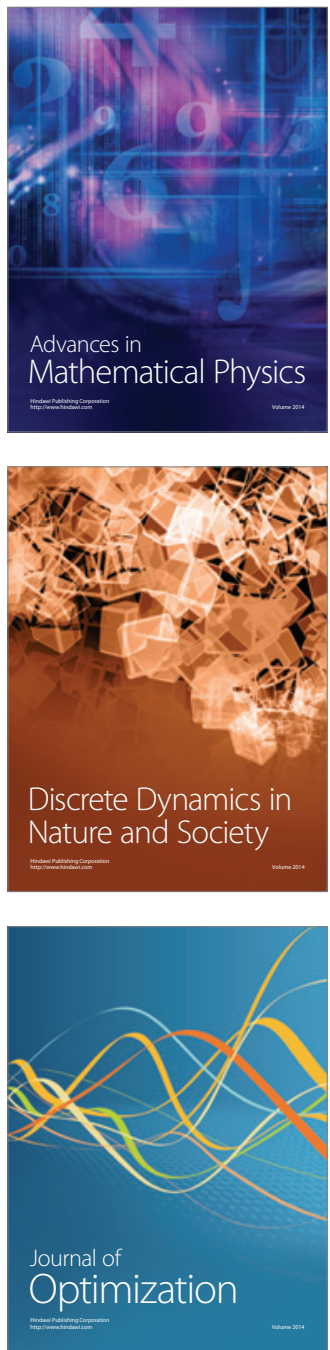